\title{
CORONAL JETS AS A CAUSE OF MICROWAVE NEGATIVE BURSTS
}

\author{
I.V. Kuzmenko \\ Institute of Applied Astronomy RAS, \\ St.Petersburg, Russia, kuzmenko_irina@mail.ru
}

\begin{abstract}
We have investigated the cause of three "isolated" negative radio bursts recorded one after another at several frequencies in the $1-17 \mathrm{GHz}$ range at the Nobeyama Radio Observatory, Ussuriysk Astrophysical Observatory, and Learmonth Solar Observatory on April 10-11, 2014. The cause of the rarely observed "isolated" negative bursts is the absorption of radio emission from the quiet Sun's regions or a radio source in the material of a large eruptive filament. Analysis of observations in different spectral ranges using images from the Nobeyama radioheliograph and the Solar Dynamics Observatory/Atmospheric Imaging Assembly has shown that the cause of all the three radio emission depressions was the screening of the limb radio source by the material of recurrent coronal jets. Parameters of
\end{abstract}

the absorbing material were estimated using a previously developed model. These estimates confirmed the absorption of solar radio emission in cold material with a temperature of $\sim 10^{4} \mathrm{~K}$ at the bottom of the jets.

Keywords: solar radio emission, microwave negative burst, radio source, active prominence, recurrent coronal jet.

\section{INTRODUCTION}

The microwave negative burst is a temporary decrease in the total microwave flux below a quasistationary level. Solar events with negative bursts are quite rare [Grechnev et al., 2013, 2018]. Most of them belong to the type of post-burst flux decrease, i.e. the emission level decreases below the pre-burst level after an impulsive burst. Such events are generally associated with the filament eruption from an active region. The cause of the negative bursts in these cases is absorption of the emission from the quiet Sun's regions and compact radio sources by low-temperature plasma of an erupting prominence [Covington, Dodson, 1953; Sawyer, 1977; Kuzmenko et al., 2009]. The area of the absorbing material is normally $<10 \%$ of the area of the solar disk, and its temperature is $\sim 10^{4} \mathrm{~K}$.

Events with isolated negative bursts are rare and therefore poorly studied. Investigation of two such events has revealed that in one case the cause of microwave depression was the absorption of radio emission from the quit Sun's regions by the erupting prominence material [Kuzmenko, Grechnev, 2017], and in another one a radio source was generally screened [Grechnev et al., 2013]. In both events, a large quiescent prominence, located outside active regions, erupted.

Besides being absorbed by the material of the erupting prominence, radio emission can also be screened by the material of small-scale plasma eruptions [Lesovoi et al., 2017; Fedotova et al., 2018; Grechnev et al., 2018], which are classified as surges (in $\mathrm{H} \alpha$ ) and jets (in extreme ultraviolet and X-ray ranges). Observations of jets in the extreme ultraviolet range have revealed both cold $\left(10^{4}-10^{5} \mathrm{~K}\right)$ and hot plasma $\left(10^{6} \mathrm{~K}\right)$ [Raouafi et al., 2016]. The lifetime of the jets is tens of minutes, which is comparable to the duration of negative radio bursts. In view of the small area of the jets, the depression of the total flux of solar radio emission can be observed if they screen a compact radio source. Another possible cause of the negative bursts is a decrease in emission from a radio source during evolution of an active region [Maksimov, Nefed'ev, 1991; Fedotova et al., 2018], although in a record of the total microwave flux such a depression due to its small scale may not manifest itself.

This paper deals with the April 10-11, 2014 solar event, during which there were three successive isolated negative bursts at a number of microwave frequencies. The purpose of the study is to find the cause of their occurrence, using a complex method of analyzing observations in different spectral ranges and a method for estimating parameters of absorbing material from observed radio absorption at different frequencies [Grechnev et al., 2008].

\section{ANALYSIS OF SOLAR OBSERVATIONS IN DIFFERENT SPECTRAL RANGES}

\subsection{Observations in a radio frequency range}

Three microwave depressions were observed on April 10-11, 2014 at a number of microwave frequencies with radio polarimeters in Nobeyama (Nobeyama Radio Observatory, Japan), Learmonth (Learmonth Solar Observatory, Australia), and Ussuriysk (Ussuriysk Astrophysical Observatory, Russia). Figure 1 shows the radio emission profiles smoothed over 20-30 s with subtracted pre-burst level $F_{\mathrm{b}}$. For most records, the preburst level was taken to be equal to the mean total flux of solar radio emission in a period 23:05-23:10 (hereinafter, Universal Time is used). Since observations at the 


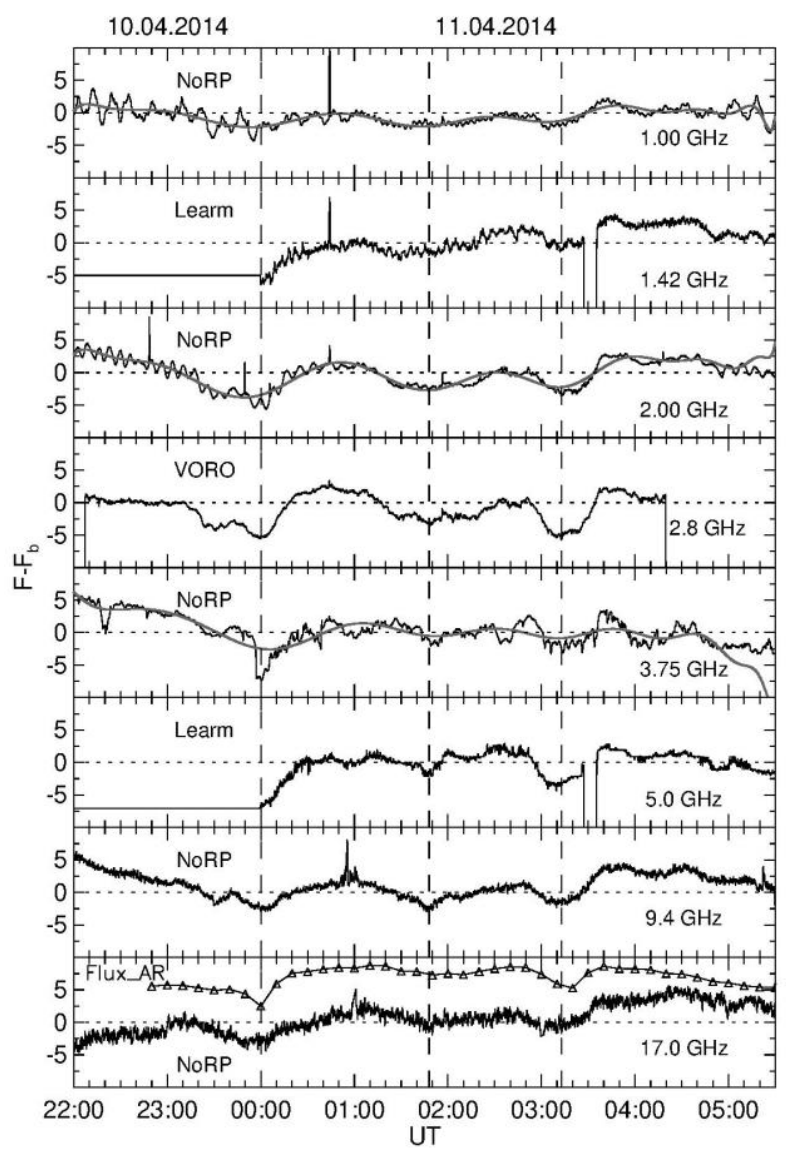

Figure 1. Radio solar emission profiles at different frequencies with the subtracted pre-burst level $F_{\mathrm{b}}$. Vertical dashed lines indicate maximum absorption depths for three microwave depressions. On the bottom panel, the curve with triangles shows a change in flux from a radio source measured from NoRH images
Learmonth Observatory started near 00:00, the mean pre-burst level for this data was calculated in a period 01:00-01:05. Flux profiles at frequencies of 1, 2, and $3.75 \mathrm{GHz}$ varied significantly, therefore they were fitted by a polynomial function (gray lines in Figure 1). The maximum depth of the negative bursts was recorded at $00: 00,1: 48$, and $3: 13$, as indicated by vertical dashed lines in Figure 1.

Since the depressions of the total radio flux were observed at a frequency of $17 \mathrm{GHz}$, which is quite rare, solar images from the Nobeyama radioheliograph (NoRH) were used to identify their possible causes. Figure 2 presents images of a part of the solar disk from observations in different spectral ranges during the microwave depression (second - fourth columns), as well as before (at 23:00, the first column on the left) and after (at 05:00, the rightmost column) recording the negative bursts.

The NoRH images at the frequency of $17 \mathrm{GHz}$ (the upper row in Figure 2) show that all the negative bursts were connected with the screening of a part of the radio source located on the eastern limb. On the bottom panel of Figure 1 above the radio flux profile at the frequency of $17 \mathrm{GHz}$ the curve with triangles indicates the emission flux from the radio source, estimated from NoRH images at $10 \mathrm{~min}$ intervals. We can see that the emission flux of the radio source varied. These variations may be associated with the evolution of an active region risingfrom behind the limb. As derived from GOES-15 data, on April 11, 2014 in this active region with coordinates $13^{\circ}-17^{\circ} \mathrm{S}, 88^{\circ} \mathrm{E}$ after the first negative burst there were C2.7 and C2.6 flares from GOES-15 data; and before the third microwave depression, C1.7 flares. Since the flares occurred on the limb, only a slight increase in the total radio flux was recorded at 00:20-01:20, without any significant impulsive bursts.

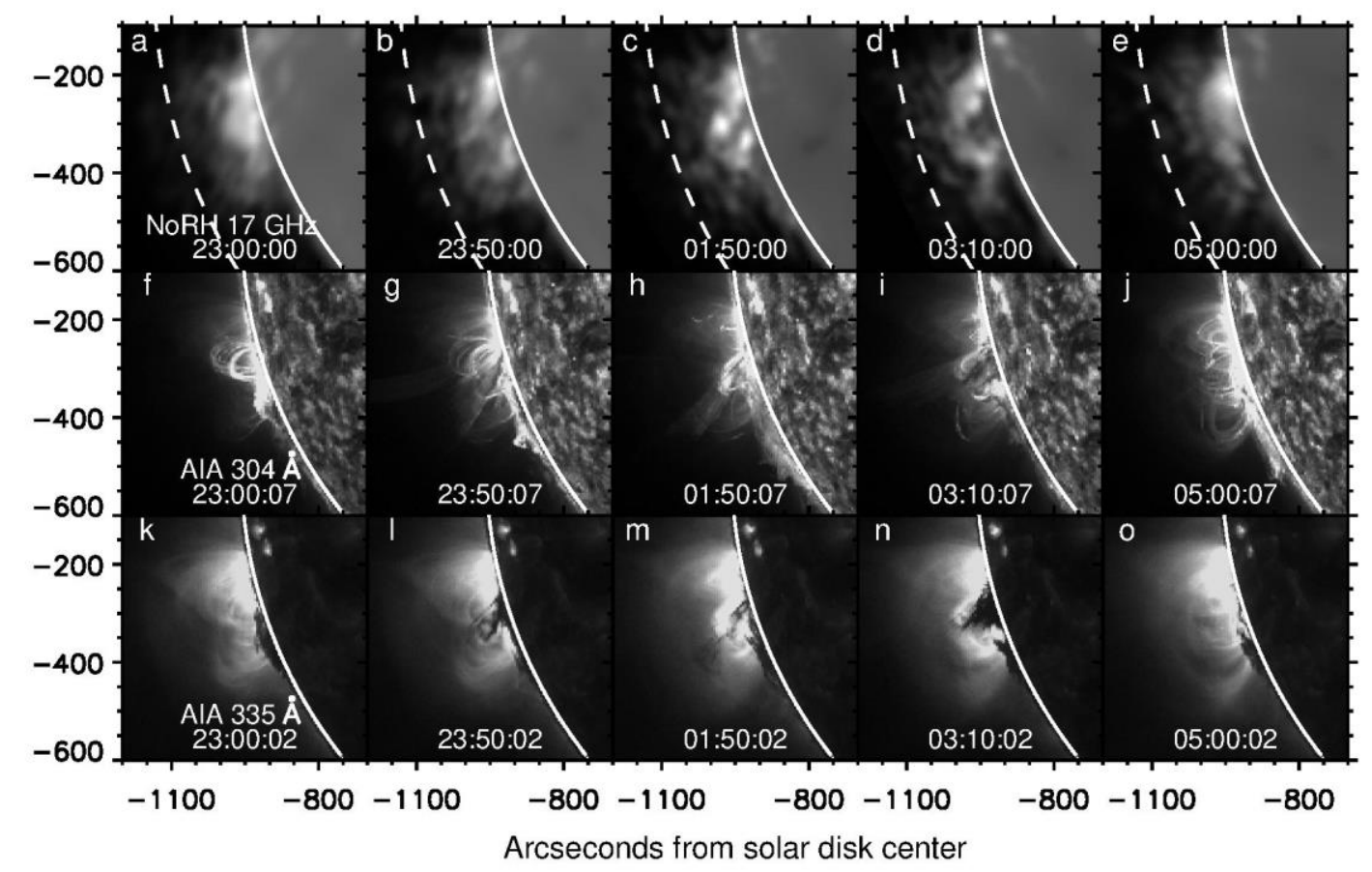

Figure 2. Images of a part of the solar disk, as derived from data obtained in different spectral ranges: the upper row $(a-e)-$ NoRH at a frequency of $17 \mathrm{GHz}$, the middle $(f-j)$ and lower $(k-o)$ rows - SDO/AIA in the 304 and $335 \AA$ channels respectively. The solid white arc marks the solar disk; the dashed arc, the solar radio radius $\left(1.186 R_{\odot}\right)$ at a frequency of $1 \mathrm{GHz}$ 
The flux decrease coincided in time with the recording of the negative bursts and with radio source screening at the frequency of $17 \mathrm{GHz}$. We can assume that the cause of all the three negative bursts was absorption of solar radio emission from the same radio source by cold material of a screen.

\subsection{Observations in other spectral ranges}

Analysis of original SDO/AIA images in the $304 \AA$ channel (a maximum temperature sensitivity of 80000 K) has revealed that on April 10-11, 2014 to the southwest of the active region a periodic activation of prominence over the limb occurred which was observed in emission (Figure 2, $g, h, j$ ). Sometimes some of its parts were dark. Note that the prominence became active between the first and second negative bursts.

During the first depression of solar radio emission from the active region, a jet was observed at 23:50-00:20 (Figure 2, g). During the second negative burst, there was also a jet (at 01:20-02:40) whose bright material included dark strips (Figure 2, $h$ ). The third microwave depression was also associated with the jet at 02:50-03:40 (Figure 2, $i$ ). The bottom of the first and third jets consisted of the darkest and densest material, and all the three jets featured backward motion of material.

The images in hot coronal channels of 211 and 335 $\AA$ (a maximum temperature sensitivity of $\sim 2 \mathrm{MK}$ ) show that the dark material periodically rose over the limb (Figure 2, $l-n$ ). There are observations from the Big Bear Solar Observatory (BBSO) in $\mathrm{H} \alpha$ on April 11, 2014 only after 17:00, but on April 10, 2014 there were surges and periodic activation of prominence on the eastern limb.

The active region near the limb could be observed in STEREO-B/EUVI images (the angle between Earth and the space observatory was $\approx 164^{\circ}$ ). There was an activating prominence and jets in the $304 \AA$ channel above the limb, and a weak jet in the $304 \AA$ channel above the limb.

A coronal mass ejection with a central position angle of $106^{\circ}$ was recorded by SOHO/LASCO on April 10, 2014 at 14:36. There was no bright core in the CME structure. On April 11, the coronagraph LASCO/C2 observed weak emerging loops to 03:12 when a brighter and denser loop appeared.

Thus, observations in different spectral ranges show the activation of prominence to the southwest of the active region, but not its eruption. The active region periodically ejected jets whose material returned to the solar surface. We can conclude that the screen that absorbed the part of the radio source emission over the eastern solar limb was the material of the recurrent jets.

\section{ESTIMATED PARAMETERS OF ABSORBING MATERIAL}

The previously developed model for estimating parameters of absorbing material from observed values of radio absorption at different frequencies [Grechnev et al., 2008; Kuzmenko et al., 2009] has been used to make such estimates for the negative bursts of interest. The model deals with the flat screen absorption of thermal emission both of the quiet Sun's regions and of the radio source on the solar disk.

In our event, the radio source was screened on the limb, but since the solar radio radius in the microwave range is larger than its optical radius, the jet material could screen not only the radio source but also small regions of the quiet Sun. Figure 2 (top row) shows the solar radio radius at the frequency of $1 \mathrm{GHz}$ (dashed arc), at which negative bursts were also detected.

The proposed model takes into account the contributions of the chromosphere and the screened radio source; of the absorbing screen with an area $A_{\mathrm{S}}$, a kinetic temperature $T_{\mathrm{S}}$, and an optical thickness $\tau_{\mathrm{S}}$, inserted into the corona at a height $h$ over the chromosphere; of the coronal layer between the screen and an observer (with an optical thickness $\tau_{2}=\tau_{\mathrm{C}} \exp (-2 h / H)$, where $H$ is the height of the uniform atmosphere; $\tau_{\mathrm{C}}$ is the optical thickness of the corona), as well as of the layer between the chromosphere and the screen (with an optical thickness $\tau_{1}=\tau_{\mathrm{C}}-\tau_{2}$ ). The optical thickness of the corona is calculated from the expression for the brightness temperature of the microwave emission of the quiet Sun $T_{\mathrm{QS}}^{\mathrm{B}} \approx T_{\mathrm{chr}}+T_{\mathrm{C}} \tau_{\mathrm{C}} ; T_{\mathrm{C}}$ and $T_{\mathrm{chr}}$ are the coronal and chromospheric temperatures respectively.

A relative decrease in the total flux of a negative burst in the case of screening of the quiet Sun's (QS) regions and a radio source (AR) by an absorbing screen:

$$
\frac{F}{F_{\mathrm{QS}}+F_{\mathrm{AR}}}=\frac{T_{\mathrm{QS}}^{\mathrm{B}}\left(A-A_{\mathrm{S}}\right)+T_{\mathrm{S}}^{\mathrm{B}} A_{\mathrm{S}}}{T_{\mathrm{QS}}^{\mathrm{B}} A+T_{\mathrm{AR}} A_{\mathrm{S}}} .
$$

Here $T_{\mathrm{QS}}^{\mathrm{B}}$ and $T_{\mathrm{S}}^{\mathrm{B}}$ are the brightness temperatures of the quiet Sun (their frequency dependences were taken from [Borovik, 1994]) and the screen respectively; $A$ is the solar disk area; $T_{\mathrm{AR}}$ is the virtual brightness temperature of the source, evenly distributed over the screen area $A_{\mathrm{S}}$, which is calculated after setting the value of $F_{\mathrm{AR}}$ from the screened radio source. The flux $F_{\mathrm{AR}}$ is usually close to flux from the source measured from NoRH images at the frequency of $17 \mathrm{GHz}$. The model considers the screening of the source emitting thermal bremsstrahlung, and the solar radio emission flux at other wavelengths is recalculated under the assumption that the screened part has the same size at different frequencies. Since in our event the active region rose from behind the limb, the possible contribution of the gyroresonance microwave radio source was considered insignificant.

The brightness temperature of the screen is

$$
\begin{aligned}
& T_{\mathrm{S}}^{\mathrm{B}}=T_{0} e^{-\left(\tau_{1}+\tau_{\mathrm{S}}+\tau_{2}\right)}+T_{\mathrm{C}} e^{-\left(\tau_{\mathrm{S}}+\tau_{2}\right)}\left(1-e^{-\tau_{1}}\right)+ \\
& +T_{\mathrm{S}} e^{-\tau_{2}}\left(1-e^{-\tau_{\mathrm{S}}}\right)+T_{\mathrm{C}}\left(1-e^{-\tau_{2}}\right) .
\end{aligned}
$$

Here, $T_{0}$ is the kinetic temperature of a screened region. In the case of screening of an optically thin source, it represents the sum of the chromospheric temperature and the virtual kinetic temperature of the source: $T_{0}=T_{\mathrm{chr}}+T_{\mathrm{AR}} e^{\tau_{\mathrm{C}}}$. If the source is optically thick, $T_{0}=T_{\mathrm{AR}}$. In our modeling, we vary the optically thin and 
thick components of radio source emission, making the curve calculated from (1) close to real measurements. Figure 3 shows normalized observed values of radio absorption (diamonds) along with the results of the model fit, represented by the solid curve for each of the three negative bursts. Measurement errors caused by instability of records of the solar radio emission flux are shown in Figure 3 by dashed vertical segments. The calculated curves have been obtained with the parameters of the absorbing material listed in Table. Each parameter was estimated by its sequential least-squares optimization. Variations in the parameters within the error ranges presented in Table and the height variation of the absorbing cloud over the chromosphere from 1 to $100 \mathrm{Mm}$ did not change the sum of the squared deviations between the fit and measurements significantly. Only a part of the radio source was screened in all the three negative bursts.

As expected, the area of the absorbing material was small $-<1 \%$ of the solar disk area; and the average temperature was $\sim 10^{4} \mathrm{~K}$. These parameters confirm the possibility that the screen that absorbed the part of the radio source emission may be the cold material at the bottom of the jets.

\section{DISCUSSION AND CONCLUSION}

The cause of the jets is considered to be the magnetic reconnection that occurs between closed lines of a new emerging magnetic flux and open lines of the surrounding magnetic field. Recurrent jets are often associated with sequential magnetic flux emergence and cancellation processes [Jiang et al., 2013].

The question about the mechanism of formation of the cold plasma component $(5-15) 10^{4} \mathrm{~K}$ of coronal jets is still open. For example, when modeling the occurrence of plasma of different temperature in jets, Yokoyama and Shibata [1995] have founded that the cold component could be formed by chromospheric plasma. Shen et al. [2012] have revealed that the cold plasma is mainly located at the base of a jet, as confirmed by observations in our event.

Chen et al. [2008, 2009] have shown that quite often near the observed jets there was a small prominence that either erupted before their emergence or gradually disappeared, delivering material to them.

The active region rising from behind the limb in the April 10-11, 2014 event developed and produced a number of $\mathrm{C}$ and $\mathrm{M}$ flares. Note that later jets continued to occur in it. Shen et al. [2017] have revealed two stages of development of the coronal jet, which were observed on April 16, 2014 in the same active region at an interval of 20 min. There were both cold and hot plasma components in these stages. At the first stage, the cause of cold plasma component might have been the magnetic reconnection that occurred in the chromosphere, and at the second stage the cold component was formed during eruption of a mini filament after emergence and subsequent cancellation of a new magnetic flux. In our case, limb observations do not discern whether the cold material observed at the base of the jets was from the northern part of the large prominence located to the southwest of the active region and delivering its material to the jets or from a mini prominence in this active region.

Another negative burst was detected at $1-5 \mathrm{GHz}$ frequencies on April 15, 2014 at 02:50-03:15. At that time,

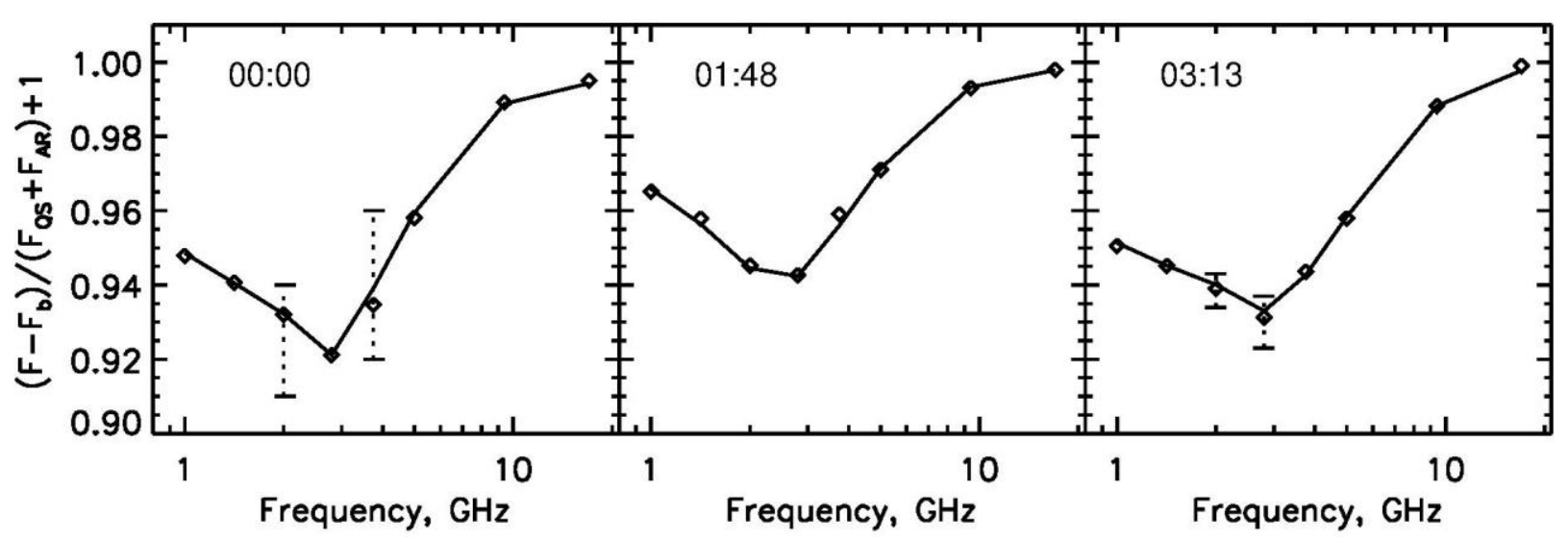

Figure 3. Spectra of the solar radio emission depth for three negative bursts: diamonds are measured values, the solid curve is the model fit. Absorption values are normalized to the sum of emission fluxes from the quiet Sun and the radio source

Absorbing material parameters estimated using the model for three depressions of solar radio emission

\begin{tabular}{|c|c|c|c|}
\hline $\begin{array}{c}\text { Depression } \\
\text { of solar radio } \\
\text { emission }\end{array}$ & $T, \mathrm{~K}$ & $\tau_{17 \mathrm{GHz}}$ & $A_{\mathrm{S}} / A_{\odot}, \%$ \\
\hline I (00:00) & $1 \cdot 10^{4} \pm 0.5 \cdot 10^{4}$ & $0.16 \pm 0.02$ & $0.4 \pm 0.1$ \\
\hline II $(01: 48)$ & $2.5 \cdot 10^{4} \pm 0.5 \cdot 10^{4}$ & $0.15 \pm 0.02$ & $0.16 \pm 0.04$ \\
\hline III $(03: 13)$ & $1 \cdot 10^{4} \pm 0.9 \cdot 10^{4}$ & $0.28 \pm 0.02$ & $0.06 \pm 0.02$ \\
\hline
\end{tabular}


SDO/AIA images in the $304 \AA$ channel showed a dark jet with the base near a sunspot in the same active region. Observations in the $\mathrm{H} \alpha$ line made at the Learmonth $\mathrm{Ob}$ servatory demonstrated small surges. There was no CME there.

Figure 4 presents a difference image of a part of the solar disk in $304 \AA$, where contour marks position of the radio source identified from NoRH images at the frequency of $17 \mathrm{GHz}$. The negative burst must have been caused by absorption of the radio source emission by the material of the jet. However, the cause might have been the screening of the gyroresonance source located above the sunspot, therefore the diagnostics of the jet plasma with the model that takes into account screening of the source emitting thermal bremsstrahlung was not made.

Note that the maximum area of the darkening in Figure 4 at a level of $25 \%$ of brightness decrease was $0.26 \%$ of the solar disk area. Grechnev et al. [2018], when studying the negative bursts caused by absorption of emission from radio sources near the solar limb by the surge material, have shown that the area of darkening on images in $304 \AA$ was $<0.2 \%$ of the solar disk area. The area of the absorbing screen is usually greater in the radio range [Kuzmenko, Grechnev, 2017], but we can hardly expect that in this case it will exceed $1 \%$.

Thus, this paper has investigated the cause of three isolated negative bursts following each other at a number of microwave frequencies on April 10-11, 2014. Analysis of solar images in different spectral ranges has revealed that all the microwave depressions occurred due to screening by the material of recurrent jets of the radio source, located on the eastern limb of the Sun. Model fits have shown that the absorbing material had a temperature of $\sim 10^{4} \mathrm{~K}$ and an area of less than $1 \%$ of the solar disk area. Observations have also confirmed that the material at the base of the jets was colder.

Analysis of the event confirmed the screening of bright near-limb radio sources by the low-temperature material of jet-like eruptions, which was detected by the Siberian radioheliograph. The screening manifested itself

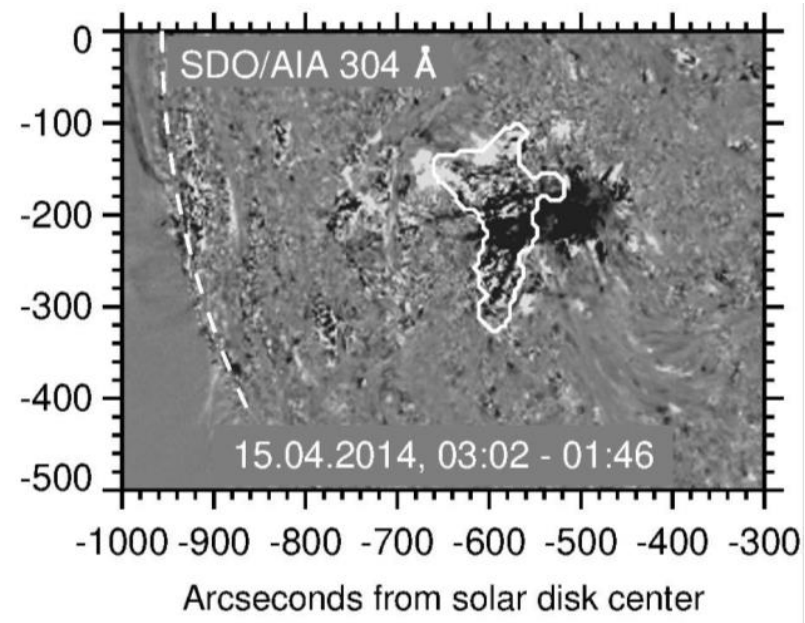

Figure 4. Difference SDO/AIA image of a part of the solar disk in the $304 \AA$ channel on April 15, 2014. The white solid contour shows position of a radio source; the dashed arc indicates the solar limb as microwave depressions on correlation plots. The isolation of the negative bursts in the event under study can be attributed to the location of the active region, where the jets occurred, exactly on the solar limb.

I thanks D.Sc. (Phys.\&Math.) Grechnev V.V. for the possibility of using programs from his library. I am grateful to teams of the Nobeyama and Learmonth observatories, SDO, SOHO, and STEREO for free access to data in different spectral ranges.

\section{REFERENCES}

Borovik V.N. Quiet Sun from the multifrequency radio observations on RATAN-600. Lectures Notes in Physics. 1994, vol. 432, pp. 185-190.

Chen H.D., Jiang Y.C., Ma S.K. Observations of H $\alpha$ surges and ultraviolet jets above satellite sunspots. Astron. Astrophys. 2008, vol. 478, pp. 907-913. DOI: 10.1051/0004-6361:20078641.

Chen H.D., Jiang Y.C., Ma S.K. An EUV jet and $\mathrm{H} \alpha$ filament eruption associated with flux cancelation in decaying active region. Solar Phys. 2009, vol. 255, iss. 1, pp. 79-90 DOI: 10.1007/s11207-008-9298-1.

Covington A.E., Dodson H.W. Absorption of 10.7-centimetre solar radiation during flare of May 19, 1951. J. Roy. Astron. Soc. Can. 1953, vol. 47, pp. 207-211.

Fedotova A.Yu., Altyntsev A.T., Kochanov A.A., Lesovoi S.V., Meshalkina N.S. Observation of eruptive events with the Siberian Radioheliograph. Solar-Terr. Phys. 2018, vol. 4, iss. 3, pp. 13-19. DOI: 10.12737/stp-43201802.

Grechnev V.V., Uralov A.M., Slemzin V.A., Chertok I.M., Kuzmenko I.V., Shibasaki K. Absorption phenomena and a probable blast wave in the 13 July 2004 eruptive event. Solar Phys. 2008, vol. 253, iss. 1-2, pp. 263-290. DOI: 10.1007/s11207-0089178-8.

Grechnev V.V., Kuzmenko I.V, Uralov A.M,, Chertok I.M., Kochanov A.A. Microwave negative bursts as indications of reconnection between eruptive filaments and large-scale coronal magnetic environment. Publ. Astron. Soc. Jap. 2013, vol. 65, no. SP1, article id. S10, 9 p. DOI: 10.1093/pasj/65.sp1.S10.

Grechnev V.V., Lesovoi S.V., Kochanov A.A., Uralov A.M., Altyntsev A.T., Gubin A.V., Zhdanov D.A., Ivanov E.F., Smolkov G.Ya., Kashapova L.K. Multi-instrument view on solar eruptive events observed with the Siberian Radioheliograph: from detection of small jets up to development of a shock wave and CME. J. Atmos. Solar-Terr. Phys. 2018, vol. 174, pp. 46-65. DOI: 10.1016/j.jastp.2018.04.014.

Jiang Y., Bi Y., Yang J., Li H., Yang B., Zheng R. Recurrent two-sided loop-type jets due to a bipole emerging below transequatorial loops. Astrophys. J. 2013, vol. 775, iss. 2, article id. 132, 6 p. DOI: 10.1088/0004-637X/775/2/132.

Kuzmenko I.V., Grechnev V.V. Development and parameters of a non-self-similar CME caused by the eruption of a quiescent prominence. Solar Phys. 2017, vol. 292, iss. 10, article id. 143, 25 p. DOI: 10.1007/s11207-017-1167-3.

Kuzmenko I.V., Grechnev V.V., Uralov A.M. A study of eruptive solar events with negative radio bursts. Astronomicheskii zhurnal [Astronomy Reports]. 2009, vol. 86, no. 11, pp. 11141124. DOI: 10.1134/S1063772909110092. (In Russian).

Lesovoi S.V., Altyncev A.T., Kochanov A.A., Grechnev V.V., Gubin A.V., Zhdanov D.A., Ivanov E.F., Uralov A.M., Kashapova L.K., Kuznecov A.A., Meshalkina N.S., Sych R.A. Siberian Radioheliograph: first results. SolarTerr. Phys. 2017, vol. 3, iss. 1, pp. 3-18. DOI: 10.12737/article_58f96ec 60fec52.86165286.

Maksimov V.P., Nefed'ev V.P. The observation of a 'negative burst' with high spatial resolution. Solar Phys. 1991, vol. 136, iss. 2, pp. 335-342. 
Raouafi N.E., Patsourakos S., Pariat E., Young P.R., Sterling A.C., Savcheva A., Shimojo M., Moreno-Insertis F., DeVore C.R., Archontis V., Török T., Mason H., Curdt W., Meyer K., Dalmasse K., Matsui Y. Solar coronal jets: observations, theory, and modeling. Space Sci. Rev. 2016, vol. 201, iss. 1-4, pp. 1-53. DOI: 10.1007/s11214-016-0260-5.

Sawyer C. Are «negative burst» due to absorption? Solar Phys. 1977, vol. 51, pp. 203-215.

Shen Y., Liu Y., Su J., Deng Y. On a coronal blowout jet: the first observation of a simultaneously produced bubble-like CME and a jet-like CME in a solar event. Astrophys. J. 2012, vol. 745 , iss. 2 , article id. 164,8 p. DOI: $10.1088 / 0004-$ 637X/745/2/164

Shen Y., Liu Y.D., Su J., Qu Z., Tian Z. On a solar blowout jet: driving mechanism and the formation of cool and hot components. Astrophys. J. 2017, vol. 851, iss. 1, article id. 67, 13 p. DOI: $10.3847 / 1538-4357 / a a 9 a 48$.

Yokoyama T., Shibata K. Magnetic reconnection as the origin of X-ray jets and H $\alpha$ surges on the Sun. Nature. 1995 , vol. 375 , iss. 6526, pp. 42-44. DOI: 10.1038/375042a0.

How to cite this article

Kuzmenko I.V. Coronal jets as a cause of microwave negative bursts. Solar-Terrestrial Physics. 2020. Vol. 6. Iss. 3. P. 23-28. DOI: $10.12737 /$ stp-63202003. 